\title{
Research on Deployment Strategies of Combine Harvesters Based on Intelligent Big Data Platform
}

\author{
Fan Zhang ${ }^{1}$, YanZhang ${ }^{2}$, HaizhaoYuan ${ }^{3}$, Chuan Sun ${ }^{4}$ and Yihang $\mathrm{Li}^{5}$ \\ ${ }^{1-2,4-5}$ School of Information Science and Technology, Agricultural University of \\ Hebei, No.289, Lingyusi Street, Baoding 071001, P. R. China. \\ ${ }^{3}$ Office of Academic Affairs, Agricultural University of Hebei \\ 1ellenzhang0911@126.com, ${ }^{2}$ zy850706@163.com, ${ }^{3}$ Piggyzhao@126.com, \\ 4840542640@qq.com, leeyihang@126.com
}

\begin{abstract}
The current agricultural machinery platforms just provide operational information of farmland and machinery, but not effective decision-making service. The problems of low utilization rate of agricultural machinery and low operation profits emerge as a major issue in the cross-regional operation of combine harvesters. The intelligent big data platform of agricultural machinery, which is firstly introduced, is not only to build an information exchanging platform for farmers and machine hand, but more important to provide the decision-making service. And then the deployment problem of combine harvesters is analyzed and the deployment model is established in the paper. Optimization deployment algorithm with global searching strategies, which is proposed in this paper, makes comparison with deployment algorithm with heuristic searching strategies that has be proposed in the author's previous article at aspects of deployment profit, cost and distances. It is concluded that the two algorithms have different applicable conditions. The better solution with high efficiency and performance can be obtained by the algorithm proposed in this paper.
\end{abstract}

Keywords: big data platform, heuristic searching, global searching, deployment strategy, combine harvester deployment

\section{Introduction}

Total powers of agricultural machinery of Hebei Province are 100 million kilowatts. the amounts of tractors and combine harvesters are 1.67 million and 127,000. The level of comprehensive agricultural mechanization is more than $70 \%$. Agricultural machinery has the characteristics of dispersing flow, seasonal operation and regulatory difficulties over a broad area. The problems of management division, information isolation, lack of scientific decision-making emerge as a major issue. At present, the existing agricultural machinery information service platforms only supply the general information of farmland and agricultural machinery for the machine hand, agricultural mechanization cooperation, but not provide effective decision-making services to obtain the maximum profits and best deployment schemes [1-4]. In the actual operation process, there are many factors that affect the allocation decision, including time, position, road condition, type and quantity, operation ability, operation price, weather and subjective factors. The machine hand has allocated combine harvesters by subjective experience without scientific and reasonable deployment plan. Too many combine harvesters crowd in some region that there is not enough farmland to reap and many of combine harvesters are idle. Other region is lack of farm machinery and need more combine harvesters to complete the harvest. The problems 
of agricultural machinery utilization decreased, cross operating income declined increasingly are more and more prominent [5-9].

Intelligence is the development trend of modern agriculture. In order to solve a series of problems such as information asymmetry, disorderly operations, the big data platform of intelligent agricultural machinery merge at a historic moment. The platform is not only to provide accurate service for machine hands, farmers, agricultural cooperatives, enterprises and other institutions, but also to provide a scientific basis for decision-making management. The effective joint between farmers and machine hands in the process of scheduling and allocating is an important means to improve the efficiency of agricultural machinery operations with the big data platform of intelligent agricultural machinery.

\section{Overview of the Big Data Platform of Intelligent Agricultural Machinery}

The construction purpose of the big data platform of intelligent agricultural machinery are as follows. Firstly, the basic information of agricultural resources is shared with the unified platform. The data, including agricultural machinery operation data, supply and demand information of operation and basic data of agricultural machinery, is acquired and fusion, analysis, open utilization the information of agricultural machinery are realized based on the agricultural data acquisition system, mobile phone APP and information management platform. Secondly, the management efficiency and the decision-making level are improved. Agricultural machinery management departments or agricultural machinery cooperation can intuitively understand the precise positioning, operation status and other related data of agricultural machinery, it makes the management decision of agricultural machinery be scientific, be intelligent, be accurate and be visual. Thirdly, information services capabilities are promoted. The information of supply and demand are provided for the machine hand, farmers, agricultural machinery cooperation. The efficiency and effectiveness of agricultural machinery operations are enhanced and depth intelligent service with intelligent scheduling, energy-saving, yield and benefit increasing are realized based on acquisition and analysis of big data, and intelligent optimization algorithm.

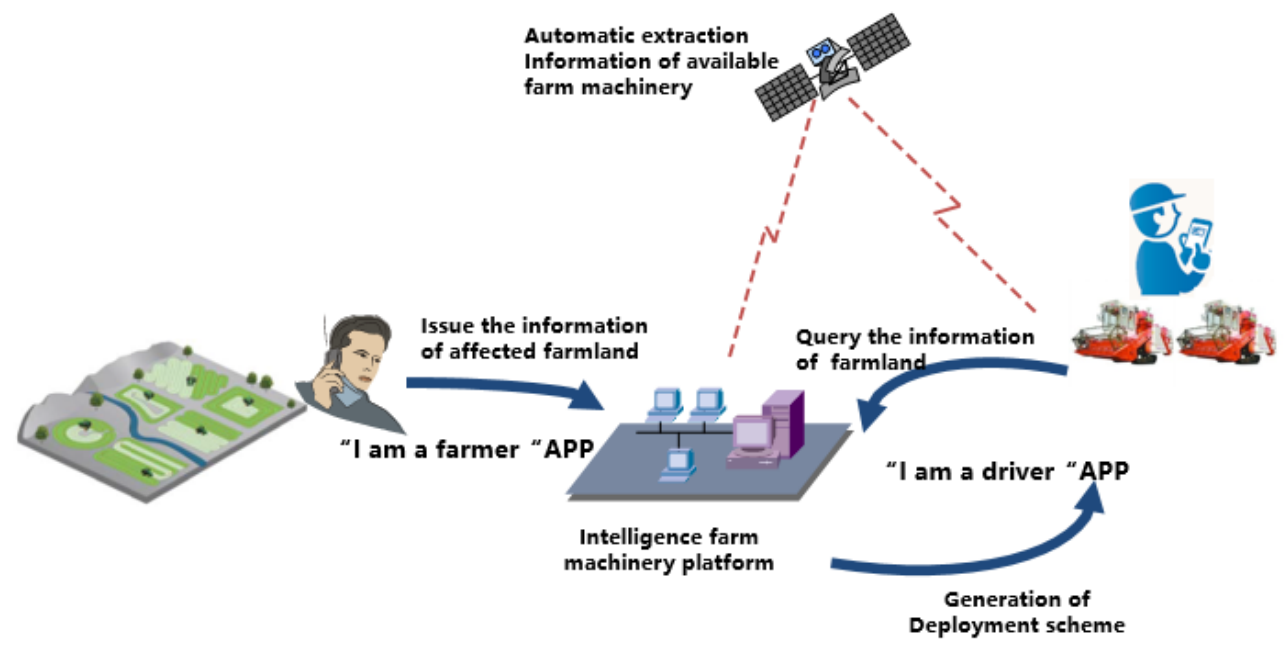

Figure 1. Schematic Diagram of Deployment of Combine Harvesters 
The main functions of the big data platform of intelligent agricultural machinery are as follows.one is the acquisition of agricultural machinery big data, and the other one is the depth service of agricultural machinery big data.

Acquisition of agricultural machinery big data. The data, which is from precise positioning, tracking, operational environment perception, is collected automatically through airborne data acquisition unit of independent research and development. The supply and demand information of farmland and agricultural machinery can be easily queried by the APP of intelligent agricultural machinery. The whole basic agricultural machinery of Hebei can be acquired by using information management platform. The data are shared among agricultural machinery management departments, enterprises, machine hands and farmers through the unified platform.

Depth service of agricultural machinery big data. Emergent scheduling and allocating system of agricultural machinery can supply the functions of information release and deployment scheme generation for agricultural machinery management department or agricultural machinery cooperation. The APPs of intelligent agricultural machinery provide optimal operation planning, information push, inquiry of supply and demand information and service information for machine hands and farmers. The depth service is realized by using two kinds of mobile phone APP. These are shown in Figure2. One is the APP of "I am a farmer" and the other one is the APP of "I am a driver".

The APP of "I am a farmer" is provide for farmers. Farmers can not only release the information of their own farmland, but also can visually browse the distribution and basic information of the surrounding available agricultural machinery through the APP. More convenience is that farmers can also make targeted selection of available farm machinery, contact machine hand directly, enjoy special combine harvester service. It can compress the operation time and improve operation efficiency and reduce losses in the harvest. The APP of "I am a driver" is provide for machine hands. Machine hands can easily browse the information of the location, areas, prices, cell phone numbers of farmers through selecting different query criteria by this APP. Machine hands can not only contact farmers directly, but also can obtain the navigation service. The APP of "I am a driver" also can provide intelligent decision service for the machine hand.
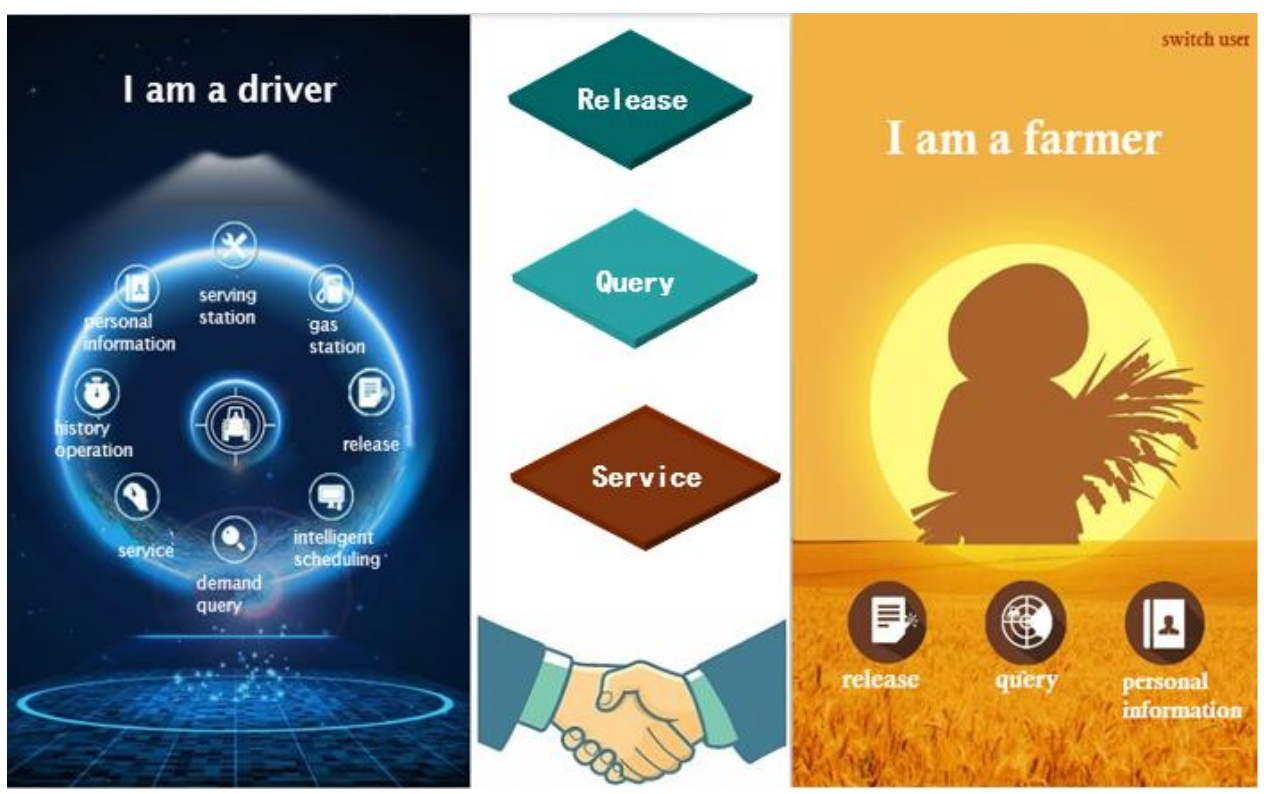

Figure 2. The Basic Functions of the Mobile APP of "I am a Driver" and "I am a Farmer" 
Considering the influence factors of the distances, area, price, operational time and weather, intelligent deployment with the goal of maximum benefit can give out top five best deployment schemes for machine hands by using efficient optimization algorithm. The functions of maintenance station, gas station and related information for the machine hand can provide quality and convenient services for smoothly operation. Intelligent deployment greatly reduces the rate of empty driving and blindness operation, and also can avoid the occurrence of problems such as waiting for the rainy day [10-15]. The using of the APPs not only can effectively save the operational cost and improve the operational efficiency of combine harvester, but also provide a strong guarantee for machine hands.

\section{Research on Deployment Strategies of Combine Harvesters.}

The big data platform of intelligent agricultural machinery is not only to build an intelligent service platform for farmers and machine hand but also to provide the best scheduling path and allocation scheme for machine hand and agricultural machinery cooperation and to achieve the goal of high yield and low cost within the time window. Intelligent planning based on big data platform of intelligent agricultural machinery is to establish a mapping relationship between multi-farmland and multi-agricultural machinery. Agricultural machinery supply services for farmland orderly through organizing appropriate route under the various constraints (such as farmland size, operational time window, weather factors). Finally, the agricultural machinery allocation goals are realized with the best deployment scheme.

To clearly describe the problem, the relevant variables of combine harvester and farmland are described in this paper. The main variables which are related to the combine harvester are defined as follows. $M_{j}$ stands for the combine harvester $\mathrm{j}$, $\mathrm{j}=1,2, \ldots \mathrm{n}$. latitude $_{j}$ is the latitude coordinates of combine harvester $j$ and longitude ${ }_{j}$

is the longitude coordinates of combine harvester $j . \not h b_{j}$ is the operation ability of combine harvester $j(\mathrm{mu} / \mathrm{h}), r v_{j}$ is the running velocity of combine harvester $j(\mathrm{Km} / \mathrm{h}), r f_{j}$ is the running fuel consumption of combine harvester $\mathrm{j}$ (yuan $/ \mathrm{km}) . w f_{j}$ is the working fuel consumption of combine harvester $\mathrm{j}(\mathrm{yuan} / \mathrm{km})$. The main variables which are related to the farmland are defined as follows. $F_{i}$ stands for the farmland $\mathrm{i}, \mathrm{i}=1,2, \ldots \mathrm{m} . S_{i}$ means the area of farmland i. latitude $i_{i}$ is the latitude coordinates of farmland $i$ and longitude $_{i}$ is the longitude coordinates of farmland $i . P_{\tilde{i}}$ is the service price of farmland i. $B_{i}$ is the suitable beginning time of farmland $\mathrm{i}$ and $E_{i}$ is the latest ending time of farmland i. The main variables, which are from combine harvesters to farmland, are defined as follows. $d_{j i}$ is the distance between combine harvester $\mathrm{j}$ and farmland i.st $t_{j i}$ is the scheduling time between combine harvester $\mathrm{j}$ and farmland $\mathrm{i} . w t_{j i}$ is the working time of combine harvester $\mathrm{j}$ serving for farmland $\mathrm{i} . a t_{j i}$ is the arrival time from combine harvester $\mathrm{j}$ to farmland i. $b t_{j i}$ is the actual beginning time of combine harvester $\mathrm{j}$ serving for farmland $i$, $e t_{j i}$ is the actual ending time of combine harvester $j$ serving for farmland $i$. Pro stands for the total profits of deployment. The decision variable is determined as $a_{j i}$, which is the allocating sign between combine harvester $j$ and farmland $i$.

$$
a_{j i}=\left\{\begin{array}{c}
1, \text { farm machinery } j \text { is allocated to farmland } i \\
0, \text { farm machinery } j \text { is unallocated to farmland } i
\end{array}\right.
$$

According to the above analysis, the intelligent deployment model is established in this paper. 


$$
\max \operatorname{Pro}=\sum_{j=1}^{n} \sum_{i=1}^{m} a_{j i} *\left(\left(P_{i} S_{i}-s t_{j i} * r f_{j i}-w t_{j i} * w f_{j i}\right)-c *\left(B_{i}-a t_{j i}\right)\right)
$$

St.

$$
\begin{gathered}
z_{j i}=\left\{\begin{array}{c}
1, B_{i}-a t_{j i}>0 \\
0, B_{i}-a t_{j i} \leq 0 \\
e t_{j i} \leq E_{i}
\end{array}\right. \\
\sum_{j=1}^{n} b b_{j} *\left(e t_{j i}-b t_{j i}\right)=S_{i} \\
b t_{j i}=\left\{\begin{array}{cc}
B_{i}, & B_{i}-a t_{j i}>0 \\
a t_{j i}, & B_{i}-a t_{j i} \leq 0
\end{array}\right\} \\
a t_{j i}=d t_{j i}+s t_{j i}
\end{gathered}
$$

From the above analysis it can be known that the goal of deployment is maximum profit of deployment. From the constraint condition (1) can see, if the arrival time $a t_{j i}$ is earlier than the suitable beginning time $B_{i}, z_{j i}=1$, otherwise, $z_{j i}=0$. From the constraint condition (2) can see, the suitable ending time of farmland i should be no more than the actual ending time. From the constraint condition (3) can see, the total completed areas of farmland $i$ are no more than the areas of i. From the constraint condition (4) can see, if the arrvial time from combine harvester $j$ to farmland $i$ is earlier than the suitabe beginning time, the actual beginning time from $\mathrm{j}$ to $\mathrm{i}$ is $B_{i}$, otherwise, the actual beginning time is $a t_{j i}$. From the constraint condition (5) can see, that the arrival time from farm machienry $j$ to farmland $i$ equals to the scheduling time from $j$ to $i$ and the completion time of farm machienry $\mathrm{j}$ serving for farmland $\mathrm{i}$.

\section{1. Scheduling and Allocating Algorithm with Global Searching Strategy}

The combine harvester scheduling and allocating problem with the constraints of time window is studied in the paper. The suitable path from the known farmland can be planned and the maximum profit can be obtained with the constraints of the time window according to the basic information of combine harvester. The specific steps of scheduling and allocating algorithm with global searching strategy are as follows.

Step1 Initial.

a. Cur points to the current farmland, cur= 0 means the location of combine harvester.

b.Next point to the next farmland which is to be searched.

c. The array stack is used for saving the searched farmland.

d.Depth stands for the depth of searching.

e. The amount of farmland is $\mathrm{m}$.

f. The upper working time limit of farmland is $\mathrm{T}$.

g. The lower profit limit of combine harvester is 0 , incomeBound $=0$.

$\mathrm{h}$. The initial profit value of combine harvester is 0 .

i. $\mathrm{k}=1$.

Step2 While $\mathrm{k}<\mathrm{m}$,turn to Step3. Otherwise, turn to Step9.

Step3 If currentIncome $>=$ incomeBound, then

depth++, stack[depth]=k, stack[depth+1]=0, Visit[k]=1;

Step4 while depth $>0$, then cur=stack[depth],next=stack[depth+1];

Step5 Search the adjacent farmland.

For $(\mathrm{i}=$ next $+1 ; \mathrm{i}<\mathrm{M} ; \mathrm{i}++)\{$

if $($ cur $==\mathrm{i})$ or visit $[\mathrm{i}]==1$ or currentTime $+\operatorname{distance}[\mathrm{cur}][\mathrm{i}] / \mathrm{v}+\mathrm{area}[\mathrm{i}] / \mathrm{p}>$ workTime 
continue;

else break;

Step6

Step 6.1

If $\mathrm{i}<\mathrm{M}$, then record the allocation of farmland $\mathrm{i}$.

a) Calculate the actual completion time of farmland i currentFinishTime[cur][i], and the profit of farmland i income[cur][i].

b) currentTime+= currentFinishTime[cur][i], currentIncome+=income[cur][i].

c) depth++.

Step 6.2

If $\mathrm{i}=\mathrm{M}$, then record the whole farmland information in this scheduling path in order.

a. maxIncome $=$ currentIncome.

b. If maxIncome $>$ incomeBound, then incomeBound= maxIncome.

c. Print out the farmland in the array stack, and obtain the scheduling and allocating scheme in order.

Step7 If depth $>0$, then

a) currentTime-= currentFinishTime [stack[depth-1]][stack[depth]].

b) currentIncome $-=$ income [stack[depth-1]][stack[depth]].

c) $\operatorname{visit}[\operatorname{stack}[\operatorname{depth}]]=0$.

d) Next=depth.

e) Depth--.

f) Return step4.

Step8 If depth $=0$, then

a) Set the array visit $[i]=0, i=\{1,2,3, \ldots, m-1\}$

b) current Time $=0$;

c) currentIncome $=0$;

d) incomeBound $=0$;

e) return Step2.

Step9 exit.

\subsection{Scheduling and Allocating Algorithm with Heuristic Searching Strategy.}

\subsubsection{Pretreatment of Farmland}

Parallel task. Each farmland has the time window of the appropriate service. $B_{i}$ and $B_{j}$ stand for the suitable beginning time of farmland $\mathrm{i}$ and $\mathrm{j}, E_{j}$ and $E_{j}$ stand for the latest ending time of farmland $\mathrm{i}$ and $\mathrm{j}$. If $B_{i}=B_{j}$ and $E_{j}=E_{j}$, the $F_{i}$ and $F_{j}$ are parallel tasks. The parallel tasks have the same layer in the paper. Therefore, the algorithm of judgment of tasks layer can be described as follows.

Step1. Initialization.

1.1 Sorts the farmland by the ascending order of earliest start time. If the farmland has the same earliest start time, sort them by the order of earliest end time and add them in the queue $Q$.

$1.2 \mathrm{n}$ is the amount of farmland. Initialize Array $\mathrm{A}[n]$, which is used to save the layer of every farmland.

1.3 The layer of $O$ is $0, j=1$.

Step2. If $j>$ n, turn to Step5.

Step3. Calculate the completion time $T_{j}$ of farmland $j$ by Multi-machinery simultaneous scheduling and allocating algorithm.

Step4. If $B_{j-1}=B_{j}$ and $E_{j-1}=E_{j}$, then the farmland $\mathrm{F}_{\mathrm{j}-1}$ and $\mathrm{F}_{j}$ have the same layer, $\mathrm{A}[j]=\mathrm{A}[j-1], j++$, turn to Step2. Else $\mathrm{A}[j]=\mathrm{A}[j-1]+1, j++$, turn to Step2.

Step5. Quit. 
The algorithm of generation of scheduling path graph can be described as follows.

Step1. Initialization.

Calculate the task amount of every layers and save in array $\mathrm{L}[\mathrm{m}] . \mathrm{m}$ is the max layer of tasks.

The layer of $O$ is $0 . \mathrm{k}=1$.

The tasks are sorted in Queue $Q$.

Step2. If $\mathrm{k}>\mathrm{m}$, turn to Step7, otherwise $\mathrm{i}=\mathrm{L}[\mathrm{k}]$.

Step3. If $\mathrm{i}>0, \mathrm{j}=\mathrm{L}[\mathrm{k}+1]$,turn to step 4 , otherwise turn to step 2.

Step4. If $\mathrm{j}>0$, turn to step5.otherwise, $\mathrm{i}--$, turn to step3.

Step5. Connect ith task of layer $i$ to jth task of layer $\mathrm{j}, \mathrm{j}--$, and turn to step4.

Step6. Sort out and generate the final scheduling path graph.

Step7. Quit.

\subsubsection{Description of the Algorithm}

In order to clearly interpret, the farmland is called task and the combine harvester held by owners is called resource.

Step1. Initialize the location of original resource is $O$ and the status of resource is 0 .

Step2. Check the task queue $Q$, if $Q$ is null, turn to Step6. Else take out the first task from the queue and turn to Step3.

Step3. Analyze the feasible resource according to the current status of resource.

Step3.1 If the resource status is 2 and its amount is not 0 , sorts these resources by the ascending order of distance from the current tasks and then calculate whether the waiting resource can complete the current tasks. If yes, turn to Step4, otherwise, calculate the completed tasks and the left tasks with the late completion time and turn to 3.2.

Step3.2 If the resource status is 1 and its amount is not 0 , sorts these resources by the ascending order of distance from the current tasks and then calculate whether the resource can complete the current tasks after releasing the previous resources. If yes, turn to Step4, otherwise, calculate the completed tasks and the left tasks with the late completion time and turn to 3.3 .

Step3.3 If the resource status is 0 and its amount is not 0 , sorts these resources by the ascending order of distance from the current tasks and then calculate whether the resource can complete the current tasks. If yes, turn to Step4, otherwise, the resource cannot complete the task and delete this task and turn to Step2.

Step4. Allocate the suitable resource for the current tasks with the lowest cost and record the allocating result.

Step5. Update the resource status of current time. Turn to Step2.

Step6. Sort out and obtain the final allocating results.

\section{Experiments and Analysis.}

The wheat harvesters from Handan city and Baoding City in Hebei Province are selected as the research object in the harvest time. These following information, which including farmland area, working ability of combine harvester and the latitude and longitude coordinates of available combine harvester and farmland, can be obtained from the experimental platform of combine harvester scheduling and allocating. The feasibility and validity of the two algorithms are further verified with the experimental data in this section. In the experiment, there are 5 groups of wheat fields and there are 6 wheat fields in each group. In order to describe clearly in the experimental parts, scheduling and allocating algorithm with global searching strategies is denoted as $A L G_{1}$ and scheduling and allocating algorithm with heuristic searching strategies is respectively denoted as $A L G_{2} . p_{1}$ and $p_{2}$ are denoted as the deployment profit of $A L G_{1}$ and $A L G_{2} . c_{1}$ and 
$c_{2}$ are denoted as the deployment cost of $A L G_{1}$ and $A L G_{2} . d_{1}$ and $d_{2}$ are denoted as the deployment distances of $A L G_{1}$ and $A L G_{2}$. The two algorithms are made a comparison at aspects of profit, cost and distances and Result Result $_{2}$, and Result $_{3}$ are defined in the following. The comparison results are as follows.

$$
\begin{aligned}
& \text { Result }_{1}=\frac{p_{1}-p_{2}}{p_{1}} \\
& \text { Result }_{2}=\frac{c_{1}-c_{2}}{c_{1}} \\
& \text { Result }_{3}=\frac{d_{1}-d_{2}}{d_{1}}
\end{aligned}
$$

Table 1. Comparison Results of Two Algorithms.

\begin{tabular}{|c|c|c|c|c|c|c|c|c|c|}
\hline \multirow{2}{*}{ NO } & \multicolumn{2}{|c|}{$\begin{array}{c}\text { Profit (ten thousand } \\
\text { yuan) }\end{array}$} & \multicolumn{2}{c|}{ Cost (ten thousand yuan) } & \multicolumn{3}{|c|}{ Distance (km) } \\
\cline { 2 - 10 } & $A L G_{1}$ & $A L G_{2}$ & Result1 & $A L G_{1}$ & $A L G_{2}$ & Result2 & $A L G_{1}$ & $A L G_{2}$ & Result3 \\
\hline 1 & 3.84 & 3.05 & $20.57 \%$ & 1.19 & 1.25 & $-5.04 \%$ & 295.15 & 315.00 & $-6.73 \%$ \\
\hline 2 & 3.15 & 2.85 & $9.52 \%$ & 1.26 & 1.37 & $-8.73 \%$ & 309.38 & 340.65 & $-10.11 \%$ \\
\hline 3 & 3.20 & 3.20 & $0.00 \%$ & 1.11 & 1.11 & $0.00 \%$ & 356.77 & 356.77 & $0.00 \%$ \\
\hline 4 & 2.94 & 2.47 & $15.99 \%$ & 1.04 & 1.16 & $-11.54 \%$ & 349.43 & 356.18 & $-1.93 \%$ \\
\hline 5 & 2.49 & 2.34 & $6.02 \%$ & 1.21 & 1.23 & $-1.65 \%$ & 406.22 & 389.75 & $4.05 \%$ \\
\hline
\end{tabular}

The results from Table 1 are shown that Result 1 is not less than $0.00 \%$, while Result2 and Result3 are not more than $0.00 \%$. it means that the total profits of $A L G_{1}$ is more than those of $A L G_{2}$, and the total costs and distances of $A L G_{1}$ is no more than those of $A L G_{2}$.That is to say, the deployment results of $A L G_{1}$ is better than those of $A L G_{2}$ from the above comparison.

To verify the feasibility and applicability of the two algorithms in depth, the comparisons are made between the two algorithms with different farm machinery amounts. The total profits of two algorithms when the amount of combine harvesters is 6,10 and 20 are shown in Figure3, Figure4 and Figure5.

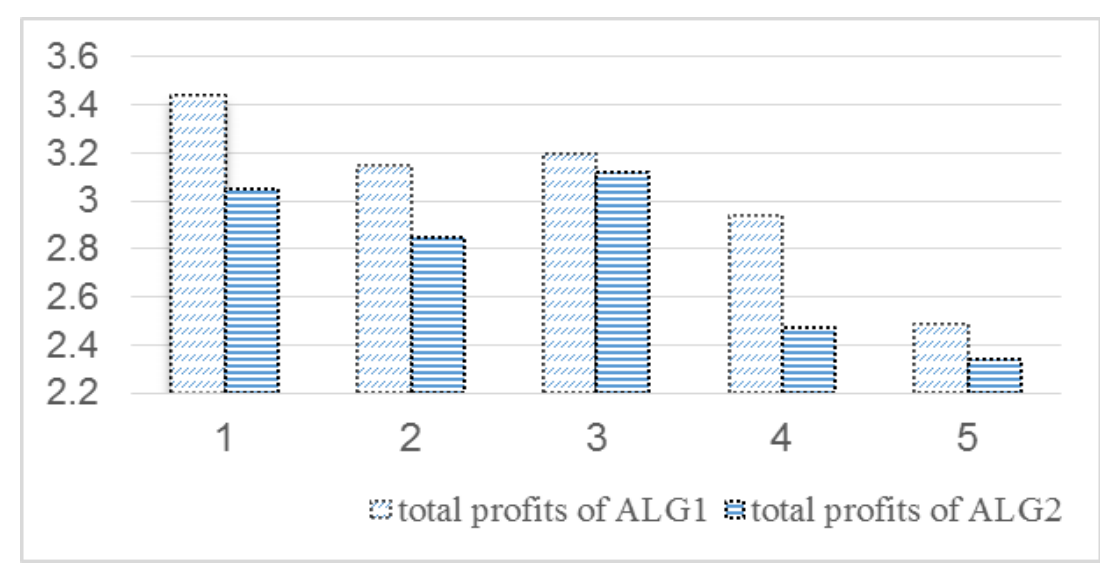

Figure 3. The Total Profits of Two Algorithms when the Amount of Combine Harvesters is 6 


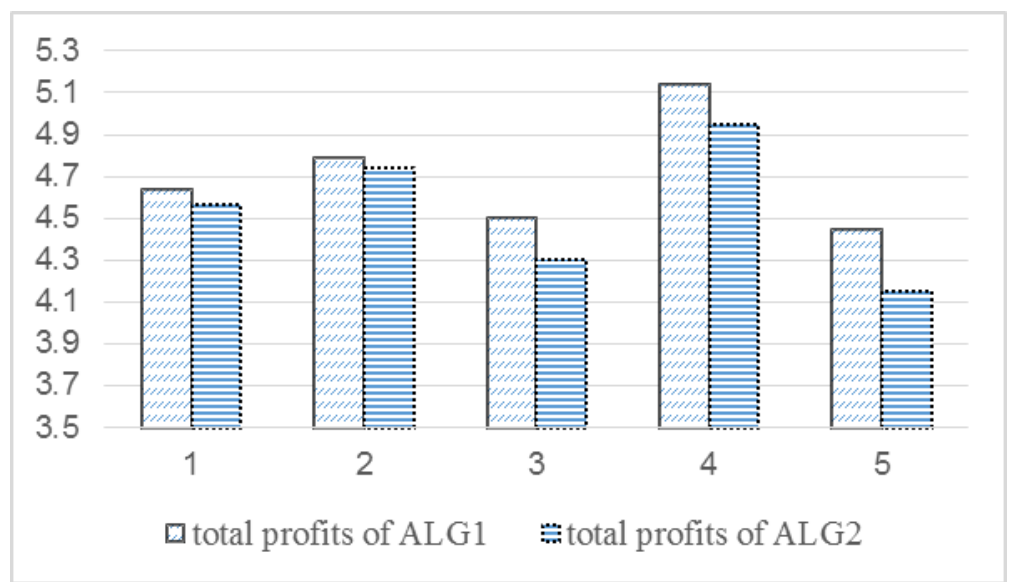

Figure 4. The Total Profits of Two Algorithms when the Amount of Combine Harvesters is 10

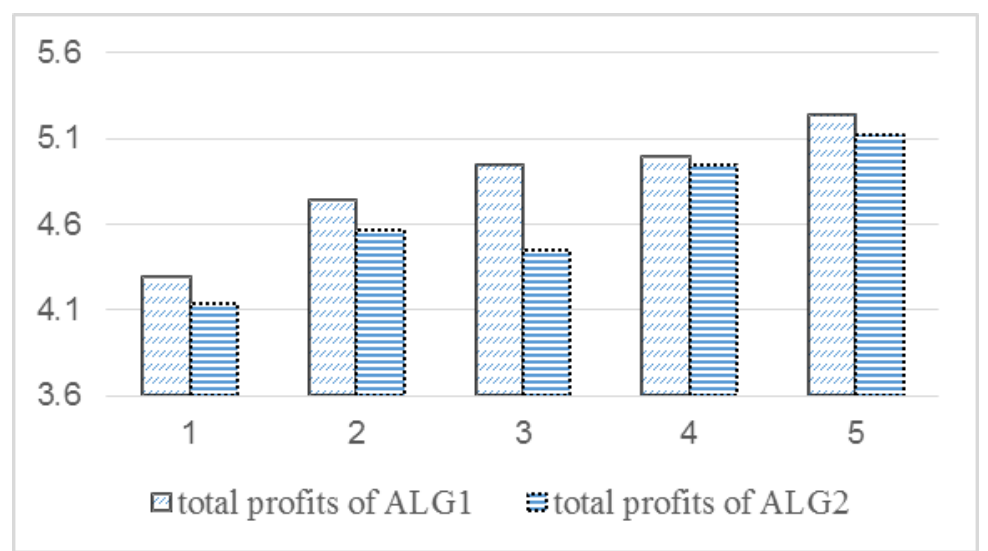

Figure 5. The Total Profits of Two Algorithms when the Amount of Combine Harvesters is $\mathbf{2 0}$

The operation efficiency of two algorithms when the amount of combine harvesters is 6,10 and 20 are shown in Figure6, Figure7 and Figure8.

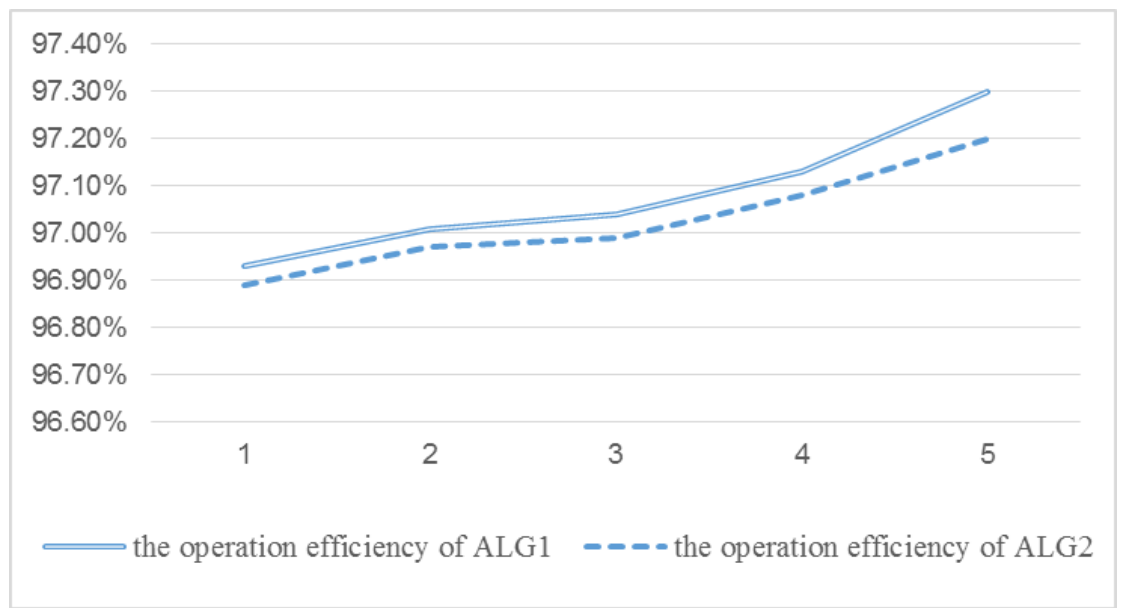

Figure 6. The Operation Efficiency of Two Algorithms when the Amount of Combine Harvesters Is 6 


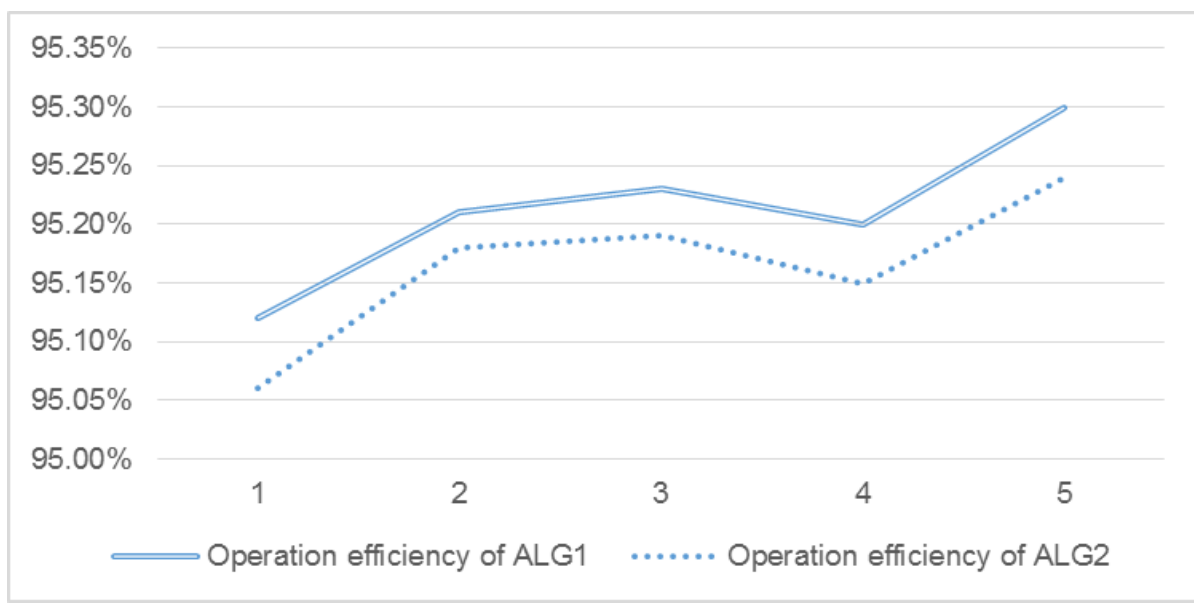

Figure 7. The Operation Efficiency of Two Algorithms when the Amount of Combine Harvesters is $\mathbf{1 0}$

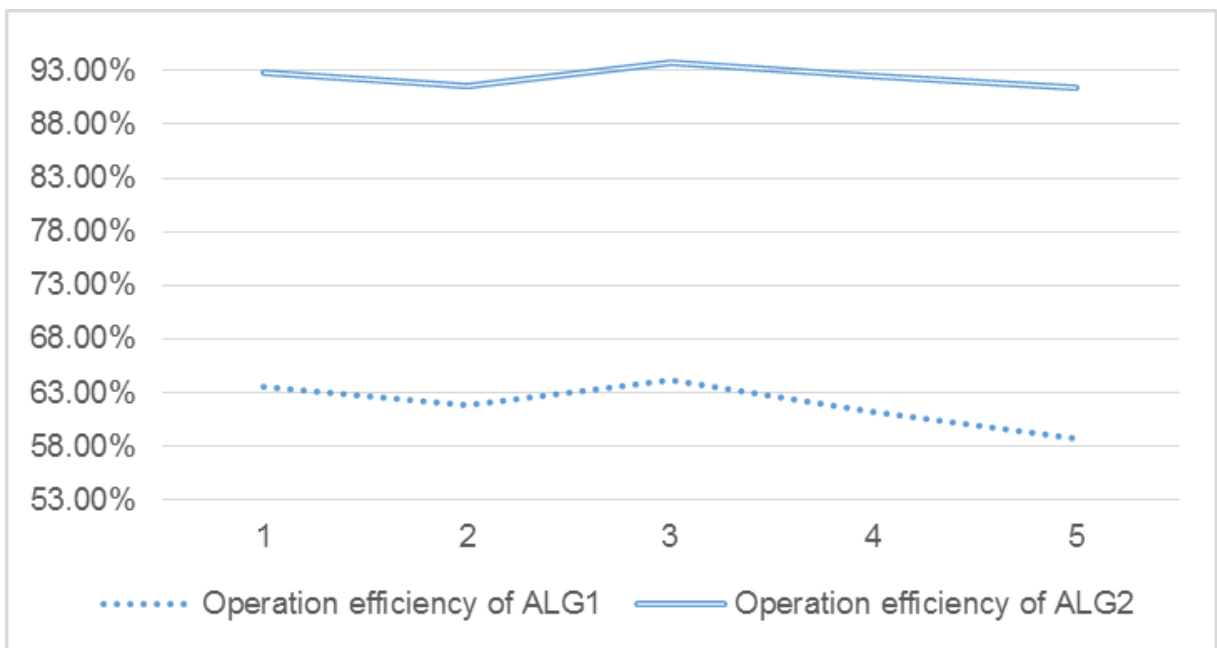

\section{Figure 8. The Operation Efficiency of Two Algorithms when the Amount of Combine Harvesters is $\mathbf{2 0}$}

From the above Figures can be seen, when the amount of combine harvesters is 6,10 and 20, the total profits of $A L G_{1}$ are a little more than those of $A L G_{2}$ in the Figure,3,Figure4 and Figure5. The operation efficiency of $A L G_{1}$ are similar to that of $A L G_{2}$ when the amount of combine harvesters is 6 and 10 from the Figure6 and Figure7. However, when the amount of combine harvesters is 20, the operation efficiency of $A L G_{1}$ is significantly smaller than that of $A L G_{2}$ in the Figure8. In conclusions, the optimum solution can be obtained by the scheduling and allocating algorithm with global searching strategies, which is a global optimal algorithm. scheduling and allocating algorithm with heuristic searching strategies, which is a local optimal algorithm, just can obtain an approximate solution. The deployment profits of local optimal algorithm are no less than those of the global optimal algorithm. The operation efficiency of the global optimal algorithm will drop considerably with increasement of problem size. 


\section{Conclusions}

The intelligent big data platform of agricultural machinery is firstly introduced, and the important function of the platform is decision-making service of deployment. The deployment problem of combine harvesters is analyzed and the deployment model is established in the paper. Optimization deployment algorithm with global searching strategies is proposed in this paper and makes comparison with deployment algorithm with heuristic searching strategies at aspects of deployment profit and operation efficiency. The deployment problem studied in the paper is based on the choice of machine hand. The amount of combine harvesters owned by machine owners are no more than 3 . Farmland has timeliness, while the overall capacity of combine harvesters is fixed. In general, the amount of farmland, which can be harvested by machine hands in the wheat harvest time, is no more than 10. Therefore, the scheduling and allocating algorithm with global searching strategies is better than the scheduling and allocating algorithm with heuristic searching strategies at the aspects of the operation efficiency and the total profits when the amount of farmland is no more than 10 .

\section{Acknowledgment}

This work is partially supported by the Key Project Fund for science and technology research of Hebei Higher Education Institutions (Grant No. Z2014168). The authors also gratefully acknowledge the helpful comments and suggestions of the reviewers, which have improved the presentation.

\section{References}

[1] Peng Bo, Design and implementation of farm SMS center farms based on GPS and GSM. Journal of China Agricultural University, (2005), Vol 2,pp70-73.

[2] Chen Quanquan, Applied research on farm machinery service information system.Science and Technology Information. (2009), Vol 13,pp14-15.

[3] Guan Congjiang, Application of digital command and control system in agricultural management.Agricultural Technology and Equipment(2009), Vol 3,pp162-163.

[4] Guo Hongpeng, L. Chen and Y. Yang, Study on the decision support system of contract hire system of agricultural machines in China.Journal of Agricultural Mechanization Research, (2006),Vol 2,pp39-42.

[5] Yu Lifeng and Xiao Xinmian, Current situation and development trend of agricultural mechanization on domestic and overseas. Journal of Hubei Agricultural Mechanization Research, (2007),Vol 6,pp36-37.

[6] Zhang Xiuhua. Study on the Service System in Trans-regional Harvesting Wheat Using Combine Harvester [D].Agricultural university of China, 2006.

[7] Yuan Yingchun,Li Xiaoping,Wang Qian. Grid Workflows Schedule Based on Priority Rules. Acta Electronica Sinica(2009),Vol 37,pp1457-1464.

[8] Buddhakulsomsiri J,Kim DS. Priority rule-based Heuristic for multi-mode resource-constrained project scheduling problems with resource vacations and activity splitting [J].European Journal of Operational Research(2007), Vol 178,pp 374-390.

[9] Liu Ming,Wu Cheng,Yang Yingjie. Genetic Algorithm Method Based on Combinatorial Rules in Identical Parallel Machine Scheduling Problem. Acta Electronica Sinica.(2000), Vol 28,pp52-54.

[10] Fan Zhang, Guifa Teng. Research on Influenced Factors about Routing Selection Scheme in Agricultural Machinery Allocation. $4^{\text {th }}$ IFIP TC 12 Conference, CCTA2010, part 2, p365-369.

[11] Fan Zhang, Guifa Teng. Research on Multitask Collaborative Scheduling Problem with Heuristic Strategies.Applied Mechanics and Materials(2011),Vol(66-68),pp758-763.

[12] Fan Zhang, Guifa Teng. Study on Farm Machinery Scheduling and Allocation Problem with Heuristic Priority Rules. ICIC Express Letters(2012),Vol 6,pp.

[13] Fan Zhang. Study on Farm Machinery Scheduling and Allocating Strategies[D].Agricultural University of Hebei,2012.

[14] Fan Zhang,Yueli Li,Chen Chen Research on Search-based Scheduling and Allocating Algorithm[J]. International Journal of Grid and Distributed ComputingVol.9, No.1, January 2016.

[15] Fan Zhang, Yuan Gao,Yueli Li.Research on Cross-Regional Emergency Scheduling and Allocating Strategies.[J] Vol.9, No.5, May 2016. 


\section{Authors}

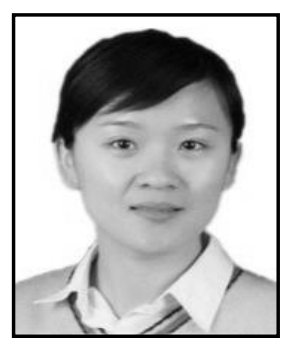

Fan Zhang, is working as a lecturer at School of Information Science and Technology, Agricultural University of Hebei. I received Ph.D. degree in 2012 and the field of research is artificial intelligence applied technology.

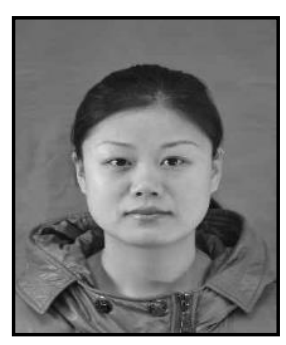

Yan Zhang, is working as a teaching assistant at School of Information Science and Technology, Agricultural University of Hebei. The field of research is agricultural informatization.

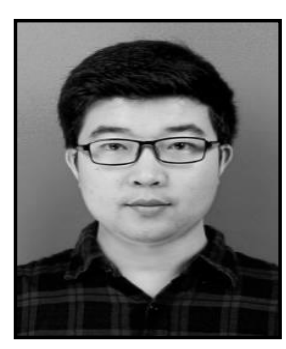

Haizhao Yuan, is working $t$ at Office of Academic Affairs, Agricultural University of Hebei. The field of research is electronic information engineering.

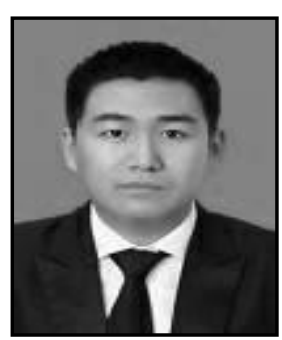

Chuan Sun, is a master Degree Candidate at School of Information Science and Technology, Agricultural University of Hebei. The field of research is artificial intelligence applied technology.

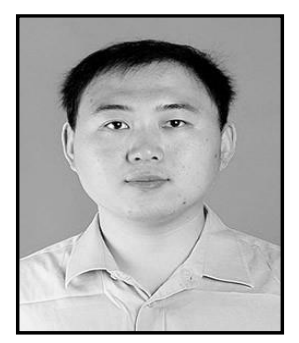

Yihang Li, is a master Degree Candidate at School of Information Science and Technology, Agricultural University of Hebei. The field of research is artificial intelligence applied technology. 